\title{
Preventing mood disorders associated with suicide- related behaviors among small- and medium-size enterprise workers in Japan
}

\section{Takaaki Fukushima}

Showa University

Osamu Takashio ( $\nabla$ takashio@med.showa-u.ac.jp )

Showa University

Akatsuki Kokaze

Showa University

Hidetoshi Sudo

Showa University

Mari Yamada

Showa University

Hideaki Kawai

Showa University

Hiromitsu Uno

Showa University

Tomohiro Yoshida

Showa University

Yukari Tosaka

Showa University

Keita Kawai

Showa University

Yukinori Kamata

Showa University

Yasuhiro Ono

Showa University

Yoshiko Oyama

Showa University

Shunsuke Hirose

Showa University

Akira Iwanami

Showa University 


\section{Research Article}

Keywords: suicide prevention, small- and medium-size enterprises in Japan, workers, anxiety disorder, mood disorder

Posted Date: March 17th, 2021

DOl: https://doi.org/10.21203/rs.3.rs-243654/v1

License: (c) (i) This work is licensed under a Creative Commons Attribution 4.0 International License.

Read Full License 
1 Preventing mood disorders associated with suicide-related behaviors among small- and

2 medium-size enterprise workers in Japan

3

4

5 Takaaki Fukushima 1,2), Osamu Takashio 1,2), Akatsuki Kokaze ${ }^{3)}$, Hidetoshi Sudo 1,2), Mari

6 Yamada 1,2), Hideaki Kawai ${ }^{1,2)}$, Hiromitsu Uno ${ }^{1,2)}$, Tomohiro Yoshida ${ }^{1,2)}$, Yukari

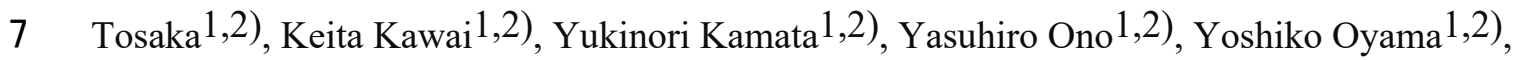

8 Shunsuke Hirose ${ }^{1,2)}$, Akira Iwanami ${ }^{1,2}$ )

9

10 1) Department of Psychiatry, Showa University School of Medicine, Setagaya-ku, Tokyo 157-

11 8577, Japan

12

13 2) Department of Psychiatry, Showa University Karasuyama Hospital, Setagaya-ku, Tokyo

14 157-8577, Japan

15

3) Department of Hygiene, Public Health and Preventive Medicine, Showa University School of Medicine, 1-5-8 Hatanodai, Shinagawa-ku, Tokyo 142-8555, Japan

\section{Corresponding author}

20 Correspondence to Osamu Takashio

\section{Abstract}

24 Background

25 While the suicide rate in Japan has recently declined, it is still high from a global perspective.

26 Recently, suicides related to unemployment caused by COVID-19 have increased. In addition,

27 because of increases in karoshi and industrial accidents, mental health measures for workers have

28 become an urgent issue. We previously conducted a study that tested whether screening for mood 29 disorders, which were strongly associated with suicide-related behavior, helps prevent suicide in 30 small and medium-size enterprise workers, where mental health support was insufficient. The 31 current study builds on the previous study's findings that mental illness screening helps identify 32 mood disorders and may provide a primary prevention method for suicide. 
A total of 1,411 consenting workers were surveyed using the Mini-International Neuropsychiatric

36 Interview (MINI). A representative module of each disease item was extracted and analyzed. The

37 Center for Epidemiological Studies-Depression (CES-D), Bipolar Spectrum Diagnostic Scale (BSDS),

38 Liebowitz Social Anxiety Scale (LSAS-J), and the Sheehan Disability Scale (SDISS) were used as

39 secondary assessments. We compared individuals with depression, bipolar disorder, dysthymia,

40 and mood disorders with a control group. In addition, we conducted multivariate analyses to

41 investigate items that identified individuals with depression, mood dysregulation, bipolar disorder, or

42 all-inclusive mood disorders.

43

$44 \quad$ Results

45 The adjusted odds ratios in the anxiety disorders groups were 3.2 for panic disorder, 2.6 for 46 social anxiety disorder, 2.1 for agoraphobia, and 1.8 for generalized anxiety disorder; 2.1 for 47 obsessive compulsive disorders; and 2.8 for the bulimia nervosa group. These results show a 48 significant correlation with mental illnesses that are referred to as neuroses in a conventional 49 diagnosis.

50

51 Conclusion

52 The study found that screening for anxiety disorders, obsessive-compulsive disorders and 53 eating disorders contributes to preventing mood disorders among workers. We hope that our 54 findings will indirectly contribute to preventing workers' suicides.

55

56 Keywords: suicide prevention, small- and medium-size enterprises in Japan, workers, anxiety 57 disorder, mood disorder

58

59

\section{Background}

60 According to the National Police Agency, deaths attributed to suicide in Japan numbered

6120,169 in 2019. According to the WHO database [1], this is the second highest suicide rate

62 among G8 countries, with Russia showing the highest rate. The number of deaths in Japan

63 attributed to suicide among employees/workers (those with a job, excluding self-employed

64 individuals and those working for a family owned company) increased two years in row up to 
65 2018. Deaths attributed to industrial accidents such as karoshi, including suicide, are also

66 increasing. The COVID-19 pandemic is expected to increase the suicide rate because of

67 increased social anxiety and a stagnant economy. In October 2020, the number of suicides per

68 month dramatically increased. One characteristic of suicide in Japan is that the rate is high

69 among young and middle age people, against a background of social and economic factors.

70 A WHO study found that 80 to $90 \%$ of those who committed suicide suffered from some

71 form of mental illness, with about $30 \%$ of those suffering from depression [1]. Studies

72 examining who attempted suicide in Japan and psychological autopsy studies are exploring

73 Japan's suicide proportions [2]. Screening for mental illness, including depression, has been

74 shown to be an important first step toward preventing suicide. Tsuchiya et al. [3] administered

75 the WHO World Mental Health Survey (WMHJ2) to assess workers' state of mental health and

76 found that the twelve-month prevalence of mental illness among workers (employees and self-

77 employed) in Japan was $2.6 \%$ for major depressive disorder, $2.3 \%$ for specific phobia, $1.6 \%$ for

78 alcohol misuse, $1.1 \%$ for social anxiety disorder (SAD), $1.0 \%$ for generalized anxiety disorder

79 (GAD), $0.6 \%$ for panic disorder (PD), $0.5 \%$ for post-traumatic stress disorder (PTSD), $0.3 \%$ for

80 type II bipolar disorder, $0.3 \%$ for mood disorder, and $0.1 \%$ for agoraphobia (AG) without PD.

81 Health surveys conducted with Japanese workers found that about $60 \%$ of the respondents had

82 severe anxiety, worries, and stress about work or professional life. This trend has continued 
83 without improvement since 1997 . More than $1 \%$ of workers suffer from mental illness and

84 continue to work while receiving medical care. Against this background, a stress check system

85 to prevent workers' mental illness was instituted in 2015 . While the system principally targets

86 primary illness prevention before onset, its secondary prevention aspect includes early

87 depression diagnosis and early treatment introduction. However, those who committed suicide

88 were unlikely to have been monitored by a medical institution. Louma et al. [4] found that more

89 than half of those who attempted suicide sought some form of medical help within a month of

90 the attempt. A project commissioned by the Ministry of Health, Labour and Welfare showed

91 that $2 \%$ of men and $5 \%$ of women who attempted suicide but survived sought help from a

92 psychiatric department before the attempt. Similar figures for seeking help from a physical

93 health department were very low, at $0.4 \%$ for men and $0.1 \%$ for women. In Japan, only one of

94 five patients with mental illness is receiving treatment [5]. To prevent suicide in Japan, we need

95 to identify hidden mental illness among the general population, including workers, and to

96 encourage them to seek help from a psychiatric specialist.

97 Unlike large companies, psychiatric support for small and medium-size enterprises has been

98 insufficient. Because of the background described above, the psychiatric field has been

99 engaging primary prevention activities as part of a suicide prevention and mental health

100 maintenance program for Japan's small- and medium-size enterprise workers since 2008. 
101 Experienced psychiatrists conduct one-to-one interviews with workers and provide information

102 and advice on stress management and sleep to prevent mental illness. If judged necessary, they

103 promptly refer patients to a specialist. We analyzed data obtained from mental health activity

104 based on our hypothesis that the key to suicide prevention is early diagnosis of mental illness,

105 including mood disorders. The results showed that, in many cases, mood disorders and anxiety

106 disorder were hidden among those who were thinking of suicide, which suggests that screening

107 for mood disorders that are strongly associated with suicide-related behavior would help prevent

108 suicides [6]. We also believe that given the frequent co-occurrence of mood and anxiety

109 disorders, identifying anxiety disorders would effectively diagnosis mood disorders for suicide

110 prevention. The National Comorbidity Survey Replication (NCS-R) in the US showed that the

111 lifetime prevalence of major depressive disorder accompanied by anxiety disorder is as high as

$11259.2 \%$. The frequency of co-occurring anxiety disorders, including SAD, single phobia, PTSD,

$113 \mathrm{GAD}$, agoraphobia, and PD (in descending order), ranged from 30 to $10 \%$ [7]. A category

114 introduced in the DSM-5, 'accompanied with anxiety-induced pain,' is thought to increase

115 suicide risk, making the illness more difficult to treat [8]. Co-occurrence of anxiety disorders

116 with bipolar disorder is also very high, with a lifetime prevalence of $74.9 \%$. Co-occurrence rates

117 (in descending order) for $\mathrm{SAD}$, single phobia, GAD, PD, obsessive compulsive disorder (OCD),

118 and PTSD range from about 40 to $10 \%$ [9]. The lifetime prevalence for persistent depressive 
119 disorder is estimated to be $1.3 \%$ in Japan [10], 2.5\% in the US [11], and around $1.5 \%$ globally

120 [12]. The co-occurrence rates in all anxiety disorder groups show a significant and positive

121 correlation [13]. These findings suggest that screening for anxiety disorders could help identify

122 mood disorders and consequently prevent suicide.

123 As far as we are aware, no other study in Japan or abroad has examined identifying mood

124 disorders and other illnesses to prevent suicide among workers. Research on the relationship

125 between mood disorders and other illnesses from the perspective of suicide prevention is also

126 limited [for example,14, 15]. Our earlier study reported that workers with mental illness and

127 suicidal ideation are hidden among small- and medium-size enterprise workers and that there

128 are many cases of mood disorder and anxiety disorder hidden among those who are suicidal.

129 This prompted us to suggest that screening for mood disorders that are strongly correlated with

130 suicide-related behavior could help prevent suicide [6]. The current study was conducted with

131 the aim of preventing suicide by screening workers for mood disorders that have a strong

132 correlation with suicide-related behavior. Since mental illness is strongly correlated with mood

133 disorders, including depression, dysthymia, and bipolar disorder, diagnosing mental illness

134 could help to prevent suicide; we therefore aimed to test the hypothesis of a correlation between

135 mood disorders and other mental illnesses among Japanese workers.

\section{Methodology}


138 The study was conducted with workers who consented to the use of data obtained from mental

139 health activities based on psychiatric health checks conducted in October and November of 2014.

\section{Research design}

141 This was a retrospective survey. To ensure anonymity, workers from multiple small- and

142 medium-size enterprises were studied.

\section{Data sources}

144 We asked research participants to complete an established psychiatric scale in addition to our

145 own questionnaire as part of a mental health activity based on a psychiatric health check. To more

146 precisely identify mental illness, we informed research participants that personal information was

147 fully protected and no information gained from the questionnaire would be passed on to their

148 employer. However, we decided to liaise with the occupational health physician or those in charge

149 of occupational health at the company if the respondent showed agitation, psychomotor agitation,

150 or urgent suicide-related behavior that was beyond the study's duty of care. However, no

151 respondents in the present study fell into this category. For this analysis, we used data from the

152 evaluation scale whose reliability and validity have been proven, but excluded responses to our

153 questionnaire.

154 Evaluation scales 
155 We used the Mini-International Neuropsychiatric Interview (MINI) as the primary evaluation

156 instrument [16]. To prioritize identifying mental illness, we selected a representative module of

157 each MINI illness item for this study.

158 For supplementary evaluation, we used the Japanese version of the Center for Epidemiological

159 Studies - Depression (CES-D) and the Japanese version of the Bipolar Spectrum Diagnostic Scale

160 (BSDS) to evaluate depression and bipolar disorder among mood disorders [17, 18]. We used the

161 Japanese version of the Liebowitz Social Anxiety Scale (LSAS-J) to assess symptoms of social

162 anxiety disorder, which is said to be prevalent among Japanese people [19]. To evaluate social

163 function and quality of life, we used the Japanese version of the Sheehan Disability Scale (SDISS)

$164[20]$.

165 Analysis methods

166 Age and the number of years of consecutive service were treated as continuous variables and

167 gender as a categorical variable. Since the MINI produces data about symptoms that are

168 characteristic of mental illness, it was used as a categorical variable. Furthermore, when either

169 depressiveness or loss of interest was checked, the participant was categorized into the depression

170 group; when either high spiritedness or increased irritability was checked, the individual was

171 categorized as the bipolar group. When either obsession or obsessive behavior was checked, the

172 individual was categorized as the obsessive compulsive disorder group, and when either of 
173 traumatic experience, a sense of powerlessness/fear or flashback was checked, the individual was

174 categorized as in the PTSD group. CES-D, BSDS, LSAS-J, and SDISS scores were treated as

175 continuous variables.

176 We then divided the research participants into two groups: a mood disorder group, including

177 depression, dysthymia, bipolar disorder, and all mood disorders and the control group. We then

178 conducted statistical analysis on survey items (gender, age, the number of years of continuous

179 service, CES-D, BSDS, LSAS-J, SDISS, and MINI other than CES-D, BSDS, LSAS-J, and

180 SDISS). Furthermore, multivariate analysis was conducted to identify items that exercise strong

181 influence on depression, dysthymia, bipolar disorder, and all mood disorders. Since there was a

182 possibility that the high correlation among depression, dysthymia, bipolar disorder, and all mood

183 disorders group could exert influence on the multivariate analysis results, we checked

184 multicollinearity using multiple regression analysis.

185 The univariate analysis statistical examination used the t-test and chi-squared test. The

186 multivariate analysis had $\mathrm{p}<0.05$ as the significance level using forced entry in the logistic

187 regression model. We used IBM SPSS Statistics ver. 22 (SPSS Inc., Tokyo, Japan) for statistical 188 analysis.

\section{$189 \quad$ Ethical considerations}


190 The study was approved by the clinical trial assessment committee of Showa University

191 Karasuyama Hospital, and confirmed that all the methods were performed in accordance with the

192 Declaration of Helsinki. Each worker was informed that personal information would be protected

193 in the study both orally and in writing and signed the informed consent document. In addition, we

194 ensured anonymity by researching several small and medium-sized enterprises. There was no

195 conflict of interest in the relationship between the research participants and their companies, the

196 third-party mediating institutions, and the researchers.

197

198 Results

199 Participant overview

200 Participant characteristics are shown in Table 1. A total of 1,411 workers (male: 1,047, female:

201364 , average age: $40.1 \pm 10.3$ years), were recruited. Most were from workplaces where the

202 number of male workers was three times that of female workers, so more men than women

203 participated. Most participants were in their forties.

204 Approximately $30 \%$ of male participants and $30 \%$ of female participants were in one of the

205 mood disorders groups. Their mean CES-D score was about 18 points and the mean LSAS-J was

206 about 47 points, which exceeded the cut-off. Their mean BSDS score was approximately 6 points. 
207 Approximately $8 \%$ of all participants were in the depression group. Their mean CES-D score

208 was about 28 points, and mean BSDS score was about 7 points. Their mean LSAS-J score was

209 high at approximately 60 points.

210 The dysthymia group accounted for about $3 \%$ of all participants. Their mean CES-D score was

211 about 30 points, and mean BSDS score was about 7 points. The mean LSAS-J score was high at

212 approximately 59 points.

213 The bipolar disorder group comprised approximately $25 \%$ of all participants. Their mean CES-

214 D score was about 17 points, and mean BSDS score was about 6 points. The mean LSAS-J score

215 was approximately 45 points.

216 There were significant between-group differences in the CES-D and LSAS-J scores; the

217 difference between the depression and dysthymia groups was more than ten points.

218 In all groups, the SDISS showed disordered communication was high in all tested situations:

219 academic/work environment, leisure/home environment, and at home. While the control group's

220 score was about 1 point, all other groups scored higher by 2 to 4 points.

221 Table 2 in the mood disorders and control groups shows the MINI results for the main

222 evaluation of identified items that correlated with other illness groups. Statistically significant

223 differences between the mood disorder group's gross odds ratios were about 9 for the panic

224 disorder group and about 6 for both the drug misuse group and obsessive-compulsive disorder 
225 group. The agoraphobia, social anxiety disorder, and generalized anxiety groups' gross odds

226 ratios were about 5. No significant difference was found in the anorexia mentalis group.

227 Table 2 in the depression and control groups shows the main MINI evaluation items'

228 correlation comparisons with other illnesses. The statistically significant gross odds ratios for the

229 depression group were about 18 for the dysthymia group, about 16 for the drug misuse group,

230 about 10 for the obsessive-compulsive disorder group, about 8 for the generalized anxiety disorder

231 and panic disorder groups, and about 7 in the social anxiety disorder group. In addition, the scores

232 for the agoraphobia, psychotic disorder, bulimia nervosa, and bipolar disorder groups were about

2333 to 5 . There was no significant difference in the alcohol misuse group.

234 Table 2 in the dysthymia and control groups shows the major MINI item comparisons with

235 other illnesses. The statistically significant gross odds ratios for the dysthymia group were about

23618 for the depression group, about 14 for the drug misuse group, about 13 for the generalized

237 anxiety disorder group, about 12 for the obsessive-compulsive disorder group, and about 7 in the

238 panic disorder group.

239 Table 2 in the bipolar disorder and control groups shows the MINI results comparisons with

240 other illnesses. The statistically significant gross odds ratios for the bipolar disorder group were

241 about 6 for the panic disorder group and about 4 for the psychotic disorder, agoraphobia, and 
242 social anxiety disorder groups. There were no significant differences in the anorexia mentalis and

243 drug misuse groups.

244 Table 3 shows the results of the multivariate analysis of the mood disorders group and other

245 illness groups. The statistically significant adjusted odds ratios were about 3.2 for the panic

246 disorder group, about 2.8 for the bulimia nervosa group, about 2.6 for the social anxiety disorder

247 group, about 2.1 for the agoraphobia and obsessive-compulsive disorder groups, about 1.8 for the

248 generalized anxiety disorder group, and about 1.7 for the PTSD group. The other items showed

249 no significant results.

250 Table 3 also shows the results of the multivariate analysis for the depression and other illness

251 groups. The statistically significant adjusted odds ratios were approximately 3.6 for the obsessive-

252 compulsive disorder group, about 2.7 for the social anxiety disorder group, about 2.7 for the

253 generalized anxiety disorder group, about 2.4 for the panic disorder group, and about 1.8 for the

254 bulimia nervosa group. No significant results were found for the other items.

255 Furthermore, Table 3 shows the results for the dysthymia group. The statistically significant

256 adjusted odds ratios were about 7 for the generalized anxiety disorder group, about 5 for the

257 obsessive-compulsive disorder group, and about 3.3 for the anorexia mentalis group. No

258 significant results were found for the other items. 
Table 3 also shows the results for the bipolar disorder group. The statistically significant

260 adjusted odds ratios were about 2.5 for the panic disorder group, 2.3 for the bulimia nervosa group,

261 about 2 for the drug misuse group, about 1.9 for the agoraphobia group, about 1.8 for the social anxiety disorder group, and about 1.7 for the PTSD group. The other items showed no significant

263 results.

264 The statistical analysis of each group showed that the variance inflation factor was below 10

265 for all groups, and there was no multicollinearity among the explanatory variables.

\section{Discussion}

268 Based on the hypothesis that screening for mental illness may provide a primary suicide

269 prevention method, we divided research participants from a previous study into mood disorders,

270 depression, dysthymia, bipolar disorder, and controls and compared their relationships with each

271 mental illness group. Our multivariate analysis showed that in the mood disorder group, the

272 relationships were about 3.2 for the panic disorder group, approximately 2.8 for the bulimia

273 nervosa group, and about 2.6 for the social anxiety disorder group. In the depression group, it was

274 about 3.6 for the obsessive- compulsive disorder group, about 2.7 for the social anxiety disorder

275 group, and about 2.7 for the generalized anxiety disorder group. In the dysthymia group, it was

276 about 7 in the generalized anxiety disorder group, about 5 in the obsessive-compulsive disorder 
277 group, and about 3.3 in the anorexia mentalis group. In the bipolar disorder group, it was about

2782.5 for the panic disorder group and about 2.3 for the bulimia nervosa group. These results suggest

279 significant correlations between mood disorder and other mental illnesses. Previous studies have

280 shown that anxiety disorders, such as panic disorder, social anxiety disorder, and generalized

281 anxiety disorder, have particularly strong correlations. This suggests that screening for anxiety

282 disorders could lead to early diagnosis and treatment of mood disorders that are associated with

283 suicide-related behavior.

284 In the panic disorder and agoraphobia groups, significant correlations were found between the

285 mood disorder, depression, and bipolar disorder groups. Although AG was previously thought to

286 be a consequence of PD, it has become an independent diagnostic category in the DSM-5. The

287 NCS-R reported that the lifetime prevalence of PD without AG is 3.7\% and that of PD with AD

288 is $1.1 \%$ [21]. The WMHJ2 in Japan reported the lifetime prevalence of PD as $0.4 \%$ for men and

$2890.7 \%$ for women, and AG without a medical history of PD as $0.3 \%$ for men and $0.5 \%$ for women.

290 Like other anxiety disorders, PD is more frequently found in women. In addition, PD tends to co-

291 occur with other, non-anxiety mental illnesses; the DSM-5 noted the possibility of PD being 'applicable to non-anxiety disorder as well as anxiety disorder.' The lifetime prevalence of depression with PD is high at 55.6\% [22]. The co-occurrence rate of bipolar disorder with PD is said to be around 20\% [23]; it has been further reported that a neurotic tendency is a clinical 
295 characteristic and anxiety sensitivity is related to severity [24]. Vignoli et al. studied workplace

296 phobic anxiety and reported that panic attacks related to the workplace or work were one

297 manifestation [25]. Since workers in the Tokyo metropolitan area commute on busy trains, we

298 believe that workers should be screened for phobic anxiety.

299 In the social anxiety disorder group, we found significant correlations with the mood disorder,

300 depression, and bipolar disorder groups. The NCS-R reported the lifetime prevalence of SAD as

$30112.1 \%$, which is the highest among anxiety disorders except for specific phobias [26]. Seventy

302 percent of cases are reported to be female; other characteristics include young, low income, low

303 academic achievement, and being single, separated, or divorced [27]. Onset is at 14.3 years on

304 average [28]. According to WMHJ2, the lifetime prevalence is $1.8 \%$ and the twelve-month

305 prevalence is $1.0 \%$. The German National Health Interview and Examination Survey (GHS-

306 MHS) reported that in $87.8 \%$ of SAD cases, one or more mental illnesses co-occurred; patients

307 had one mental illness $20 \%$ of cases, two mental illnesses in $20 \%$ of cases, and three or more

308 mental illnesses in $60 \%$ of cases. SAD is often accompanied by other anxiety disorders, with

309 localized phobia, PD, and GAD found in approximately 20 to $30 \%$ of cases. Apart from anxiety

310 disorder, the mood disorder group comprised $65.3 \%$; $50.5 \%$ of which had major depressive

311 disorder and 38.1\% had dysthymia [29]. In many cases, SAD appears before other illnesses; when

312 accompanied by depression, suicidal ideation becomes more frequent [30]. Even when not 
313 accompanied by depression, many cases of anxiety disorder lead to suicide [31]. Compared to

314 those with other anxiety disorders, workers with SAD perform significantly worse in the

315 workplace, and are twice as likely to be expected to work even if they are unemployed. It is

316 expected that their job performance skills are maintained except in a socializing context [32]. As

317 taijin kyofusho is globally known as an illness that is peculiar to Japanese culture, it is likely that

318 SAD is hidden among many workers in Japan.

319 In the generalized anxiety disorder group, significant correlations were found with the mood

320 disorder, depression, and dysthymia groups. The lifetime prevalence of GAD is reported to be

$3215.8 \%$, with a twelve-month prevalence of 3.1\% according to NCS-R [13]. WMHJ2 reported the

322 prevalence as $1.6 \%$ and $0.6 \%$, respectively. Only $30 \%$ of individuals with GAD consulted with

323 psychiatric professionals, and only $18 \%$ consulted with medical professionals, including

324 psychosomatic medical professionals. Many individuals with anxiety disorders do not receive

325 medical treatment, and this is not limited to GAD [10]. Some sufferers consult with experts in

326 internal, cardiovascular, respiratory, or gastroenterological medicine chiefly complaining of

327 physical pain [33]. Progress is chronic and the condition can worsen with deterioration of family

328 relationships, co-occurrence of cluster C personality disorder, or an I-axis disorder [34]. Studies

329 have shown that GAD precedes depression and predicts its onset; contributes to increased

330 depression; and increases the risk of suicide attempts [35]. Therefore, intervention at an early 
331 stage is important [36]. GAD is often accompanied by other illnesses, and their lifetime co-

332 occurrence rate could reach 90\%, including depression (62.4\%), dysthymia (39.5\%), alcohol

333 dependency (37.6\%), and PD (23.5\%) [33]. While our study did not find a significant result, the

334 co-occurrence of bipolar disorder and GAD is reported to be about 12\% [37]. Our results showed

335 a strong correlation between the depression group, which is estimated to be hidden among workers

336 with chronic anxiety related to the work environment, and the dysthymia group; therefore, we

337 regard GAD prevention as important for preventing suicide.

338 In the obsessive-compulsive disorder group, we found significant correlations with the mood

339 disorder, depression, and dysthymia groups. Although there are no large-scale epidemiological

340 data in Japan, the prevalence rate is estimated to be $1 \%-2 \%$. The lifetime prevalence of mental

341 illnesses accompanying OCD has been reported as major depressive disorder (65.9\%), dysthymia

342 (24.0\%), SAD (23.4\%), PD (23.4\%), GAD (18.3\%), AG (17.7\%), bipolar disorder (12.3\%), and

$343 \mathrm{BN}(9.6 \%)$ [38]. Major depressive order is very frequent, which could be explained by

344 psychological conflict and mental fatigue related to OCD or functional problems in everyday or

345 social life. A survey of workers with OCD with hoarding symptoms showed that more severe

346 hoarding symptoms are associated with more severe problems at work because symptoms are

347 directly linked to problems at the workplace. Moreover, troubled relationships with colleagues and a lack of concentration leads to problems in daily life and work performance [39]. Our study 
349 did not find any significant correlation between OCD and bipolar disorder; their co-occurrence

350 rate has been reported as 10 to $20 \%$ of bipolar disorder cases [40]. Screening for obsessive-

351 compulsive disorder could prevent workers from developing depression because they are

352 exhausted by excessive confirmatory work. In the PTSD group, significant correlations were

353 found between the mood disorder and bipolar disorder groups. The lifetime prevalence of PTSD

354 in Japan is reported to be $0.7 \%$, with a twelve-month prevalence of $1.3 \%$ [41]. Since it is unlikely

355 that many employers are aware of PTSD among workers, we need to start with education.

356 In the anorexia mentalis group, significant correlations were found with the dysthymia group

357 and the bulimia nervosa group showing correlations with the mood disorder, depression, and

358 bipolar disorder groups. The Ministry of Health, Labour and Welfare reported that in 2016 there

359 were approximately 210,000 individuals with ED in Japan. The number of individuals with BN

360 is reported to be just below 40,000. Early intervention is important for workers who manage stress

361 through eating behavior.

362 In the drug misuse group, significant correlations were found with the bipolar disorder group.

363 A Ministry of Health, Labour and Welfare study reported that the co-occurrence rate of mood

364 disorder not directly triggered by drug misuse was $9.6 \%$, which is the second highest rate,

365 following anxiety disorder/neurosis at $12.9 \%$. Although we do not expect to find many drug

366 abusers among workers, this illness cannot be overlooked in screening. 
367 SAD, AG, PG, and GAD, which are conventionally classified as neuroses, are reorganized as

368 'anxiety disorder' in the DSM-5, and are now in a single category with separation anxiety and

369 akinetic mutism. OCD has been reclassified as an independent category of 'obsessiveness and its

370 related disorder' and PTSD and acute stress disorder are now classified as 'trauma and stress

371 related disorder' together with adaptive disorder. Our findings suggest that mental illnesses that

372 fit the classical sense of neurosis have significantly strong correlations with mood disorders. Since

373 anxiety disorder generally has an early onset [28], it is said that mood disorders lead to anxiety

374 disorders. If this is the case, the illness is likely to become severe and chronic, increasing the

375 suicide risk. Workers' anxiety disorders can worsen through their work and become work

376 performance obstacles, which can then induce mood disorders, further complicating the illness.

377 Since OCD, PTSD, and ED are classified as neuroses in the bigger scheme, identifying workers'

378 mental illnesses seen as neuroses in a conventional sense may suppress the onset of mood

379 disorders and contribute to preventing suicide.

380 One limitation of the current study is that it focused on white-collar male workers in the Tokyo

381 metropolitan area; therefore, the findings cannot be broadly generalized. In addition, because we

used a self-administered evaluation scale, diagnosis and evaluation were limited. Furthermore, we did not conduct a longitudinal evaluation. Since mental illness diagnoses could change through the course of development, it is necessary to monitor progress over the long term. Wang et al. 
385 [42] investigated sleep disorders as a mental illness related to suicidal risk and reported that

386 nightmares and insomnia could increase the suicide risk in patients with depression. This and

387 other similar illnesses need to be the focus of future research.

\section{Conclusion}

389 The present study findings suggest that screening workers for mental illnesses that fall under

390 the conventional category of neurosis, such as anxiety disorder, may help to prevent the onset of

391 mood disorders and suicide. It is hoped that more research will be conducted to help prevent

392 suicide, and to enhance economic activity by identifying mental illnesses hidden among

393 workers.

394

395 Abbreviations

396 WHO: World Health Organization

397 WMHJ2: The World Mental Health Survey Second

398 SAD: Social anxiety disorder

399 GAD: Generalized anxiety disorder

400 PD: Panic disorder

401 PTSD: Post traumatic stress disorder

402 AG: Agoraphobia

403 NCS-R: National Comorbidity Survey Replication

404 OCD: Obsessive-compulsive disorder

405 MINI: The Mini-International Neuropsychiatric Interview

406 CES-D: Center for Epidemiological Studies-Depression

407 BSDS: Bipolar Spectrum Diagnostic Scale

408 LSAS-J: Liebowitz Social Anxiety Scale

409 SDISS: Sheehan Disability Scale

410 GHS -MHS: German National Health Interview and Examination Survey

411 BN: Bulimia nervosa 
412 ED: Eating disorders

413

414 Declarations

415

416 Ethics approval and consent to participate

417 The study was approved by the clinical trial assessment committee of Showa University

418 Karasuyama Hospital, and confirmed that all the methods were performed in accordance with

419 the Declaration of Helsinki. Each worker was informed that personal information would be

420 protected in the study both orally and in writing and signed the informed consent document. In

421 addition, we ensured anonymity by researching several small and medium-sized enterprises.

422 There was no conflict of interest in the relationship between the research participants and their

423 companies, the third-party mediating institutions, and the researchers.

424

425 Consent for publication

426 Not applicable.

427

428 Availability of data and materials

429 The data are available from the corresponding authors upon reasonable request.

430

431 Competing interests

432 The authors declare that they have no competing interests.

433

$434 \quad$ Funding

435 No funding was provided for this study.

436

437 Authors' contributions

$438 \mathrm{TF}$ and OT designed the study, performed statistical analysis, interpreted the data, and wrote the 439 paper. HS, MY, HK, HU, and TY participated in the study conception. YT, KK, YK, YO, YO, 440 and SH collected, entered, and organized data. AK managed and conducted the statistical

441 analyses and checked the final manuscript, especially with regard to data. AI revised the

442 manuscript to develop the final manuscript. All authors have approved the final manuscript.

443

444 Acknowledgements

445 Mr. AZEKAWA Kazuhiro, Ms. IRIE Mari, and Ms. OOBA Rie of MHI Co., Limited, have

446 helped us tremendously. We would like to deeply thank them for their support in protecting the

447 personal information of research participants in the current study and in all research. 


\section{References}

451

452

453

454

455

456

457

458

459

460

461

462

463

464

465

466

467

468

469

470

471

472

473

474

475

476

477

478

479

480

481

482

483

1. World Health Data Platform / GHO $>$ Themes $>$ Mental health $>$ Suicide data https://www.who.int/teams/mental-health-and-substance-use/suicide-data Accessed 20 Dec 2020.

2. Asukai N. [Mental disorder as a risk factor of suicide; a clinical study of failed suicides]. Seishin Shinkeigaku Zasshi. 1994;96(6):415-43. Japanese. PMID: 7938308.

3. Tsuchiya M, Kawakami N, Ono Y, Nakane Y, Nakamura Y, Fukao A, Tachimori H, Iwata N, Uda H, Nakane H, Watanabe M, Oorui M, Naganuma Y, Furukawa TA, Kobayashi M, Ahiko T, Takeshima T, Kikkawa T. Impact of mental disorders on work performance in a community sample of workers in Japan: the World Mental Health Japan Survey 2002-2005. Psychiatry Res. 2012 Jun 30;198(1):140-5. doi: 10.1016/j.psychres.2011.10.014. Epub 2012 Feb 27. PMID: 22374551.

4. Luoma JB, Martin CE, Pearson JL. Contact with mental health and primary care providers before suicide: a review of the evidence. Am J Psychiatry. 2002 Jun;159(6):909-16. doi: 10.1176/appi.ajp.159.6.909. PMID: 12042175; PMCID: PMC5072576.

5. Ishikawa H, Kawakami N, Kessler RC. World Mental Health Japan Survey Collaborators. Lifetime and 12-month prevalence, severity, and unmet need for treatment of common mental disorders in Japan: results from the final dataset of the World Mental Health Japan Survey. Epidemiol Psychiatr Sci. 2016 Jun;25(3):217-29. doi: 10.1017/S2045796015000566. Epub 2015 Jul 7. PMID: 26148821; PMCID: PMC5144586.

6. Funako T, Takashio O, Igarashi R, Harada A, Ohta M, Omori Y, Sato A, Sawanobori Y, Toki T, Nakamur Y, Fukushima T, Shimizu H, Sanada K, Yamada H, Iwanami A, Kawaguchi Y, Minegishi G. Suicide prevention for Japanese employees at small and medium-sized enterprises using the Showa university style psychiatric health check-up. Journal of The Showa University Society. 2018;78:38-47. https://doi.org/10.14930/jshowaunivsoc. 78.38

7. Kessler RC, Berglund P, Demler O, et al. The Epidemiology of Major Depressive Disorder: Results from the National Comorbidity Survey Replication (NCSR) JAMA. 2003;289(23):3095-105. doi:10.1001/jama.289.23.3095

8. Fava M, Rush AJ, Alpert JE., et al., Difference in treatment outcome in outpatients with anxious versus nonanxious depression: a STAR*D report. Am J Psychiatry. 2008;165: 342-51. https://doi.org/10.1176/appi.ajp.2007.06111868 
484

485

486

487

488

489

490

491

492

493

494

495

496

497

498

499

500

501

502

503

504

505

506

507

508

509

510

511

512

513

514

515

516

517

518

9. Merikangas KR, Akiskal HS, Angst J et al. Lifetime and 12-month prevalence of bipolar spectrum disorder in the National Comorbidity Survey replication. Arch Gen Psychiatry. 2007;64(5):543-552. doi:10.1001/archpsyc.64.5.543

10. Ishikawa H, Kawakami N, Kessler RC. World Mental Health Japan Survey Collaborators. Lifetime and 12-month prevalence, severity, and unmet need for treatment of common mental disorders in Japan: results from the final dataset of the World Mental Health Japan Survey. Epidemiol Psychiatr Sci. 2016 Jun;25(3):217-29. doi: 10.1017/S2045796015000566. Epub 2015 Jul 7. PMID: 26148821; PMCID: PMC5144586.

11. Kessler RC, Berglund P, Demler O, Jin R, Merikangas KR, Walters EE. Lifetime prevalence and age-of-onset distributions of DSM-IV disorders in the National Comorbidity Survey Replication. Arch Gen Psychiatry. 2005 Jun;62(6):593-602. doi: 10.1001/archpsyc.62.6.593. Erratum in Arch Gen Psychiatry. 2005 Jul;62(7):768.

12. Vos T. Years lived with disability (YLDs) for 1160 sequelae of 289 diseases and injuries 1990-2010: a systematic analysis for the Global Burden of Disease Study 2010. Lancet. 2012;380(9859): 2163-96.

13. Kessler RC, Chiu WT, Demler O, Merikangas KR, Walters EE. Prevalence, severity, and comorbidity of 12-month DSM-IV disorders in the National Comorbidity Survey Replication. Arch Gen Psychiatry. 2005;62(6):617-27. doi: 10.1001/archpsyc.62.6.617. Erratum in Arch Gen Psychiatry. 2005;62(7):709.

14. Ono Y, Awata S, Iida H, Ishida Y, Ishizuka N, Iwasa H, Kamei Y, Motohashi Y, Nakagawa A, Nakamura J, Nishi N, Otsuka K, Oyama H, Sakai A, Sakai H, Suzuki Y, Tajima M, Tanaka E, Uda H, Yonemoto N, Yotsumoto T, Watanabe N. A community intervention trial of a multimodal suicide prevention program in Japan: a novel multimodal community intervention program to prevent suicide and suicide attempt in Japan, NOCOMIT-J. BMC Public Health. 2008;8:315. doi: 10.1186/1471-2458-8-315. PMID: 18793423; PMCID: PMC2551615.

15. Hirayasu Y, Kawanishi C, Yonemoto N, Ishizuka N, Okubo Y, Sakai A, Kishimoto T, Miyaoka H, Otsuka K, Kamijo Y, Matsuoka Y, Aruga T. A randomized controlled multicenter trial of post-suicide attempt case management for the prevention of further attempts in Japan (ACTION-J). BMC Public Health. 2009;9:364. doi: 10.1186/14712458-9-364. PMID: 19781096; PMCID: PMC2760885

16. Sheehan DV, Lecrubier Y, Sheehan KH, Amorim P, Janavs J, Weiller E, Hergueta T, Baker R, Dunbar GC. The Mini-International Neuropsychiatric Interview (M.I.N.I.): The development and validation of a structured diagnostic psychiatric interview for 
519

DSM-IV and ICD-10. J Clin Psychiatry. 1998;59 Suppl 20:22-33;quiz 34-57. PMID: 9881538.

17. Radloff LS. The CES-D Scale: a self-report depression scale for research in the general population. Appl Psycol Meas. 1977;1:385-401. https://doi.org/10.1177/014662167700100306

18. Nassir Ghaemi S, Miller CJ, Berv DA, Klugman J, Rosenquist KJ, Pies RW. Sensitivity and specificity of a new bipolar spectrum diagnostic scale J Affect Disord. 2005 Feb;84(2-3):273-7. doi: 10.1016/S0165-0327(03)00196-4. PMID: 15708426.

19. Liebowitz MR. Social phobia. Mod Probl Pharmacopsychiatry 1987;22:141-73. doi: 10.1159/000414022. PMID: 2885745.

20. Sheehan DV, Harnett-Sheehan K, Raj BA. The measurement of disability. Int Clin Psychopharmacol. 1996 Jun;11 Suppl 3:89-95. doi: 10.1097/00004850199606003-00015. PMID: 8923116.

21. Kessler RC, Chiu WT, Jin R, Ruscio AM, Shear K, Walters EE. The epidemiology of panic attacks, panic disorder, and agoraphobia in the National Comorbidity Survey Replication. Arch Gen Psychiatry. 2006;63(4):415-424. doi:10.1001/archpsyc.63.4.415

22. Kessler RC, Stang PE, Wittchen H, Ustun TB, Roy-Burne PP, Walters EE. Lifetime Panic-Depression Comorbidity in the National Comorbidity Survey. Arch Gen Psychiatry. 1998;55(9):801-808. doi:10.1001/archpsyc.55.9.801

23. Birmaher B, Kennah A, Brent D, Ehmann M, Bridge J, Axelson D. Is bipolar disorder specifically associated with panic disorder in youths? J Clin Psychiatry. 2002 May;63(5):414-9. doi: 10.4088/jcp.v63n0507. PMID: 12019666.

24. Simon NM, Otto MW, Fischmann D, Racette S, Nierenberg AA, Pollack MH, Smoller JW. Panic disorder and bipolar disorder: anxiety sensitivity as a potential mediator of panic during manic states. J Affect Disord. 2005 Jul;87(1):101-5. doi: 10.1016/j.jad.2005.02.004. PMID: 15894380.

25. Vignoli M, Muschalla B, Mariani MG. Workplace Phobic Anxiety as a Mental Health Phenomenon in the Job Demands-Resources Model Biomed Res Int. 2017;2017:3285092. doi: 10.1155/2017/3285092. Epub 2017 Nov 29. PMID: 29318143; PMCID: PMC5727632.

26. Kessler RC, Berglund P, Demler O, Jin R, Merikangas KR, Walters EE. Lifetime prevalence and age-of-onset distributions of DSM-IV disorders in the National Comorbidity Survey Replication. Arch Gen Psychiatry. 2005 Jun;62(6):593-602. doi: 10.1001/archpsyc.62.6.593. Erratum in Arch Gen Psychiatry. 2005 Jul;62(7):768. 
554

555

556

557

558

559

560

561

562

563

564

565

566

567

568

569

570

571

572

573

574

575

576

577

578

579

580

581

582

583

584

585

586

587

588

589

27. Schneier FR, Johnson J, Hornig CD, Liebowitz MR, Weissman MM. Social Phobia: Comorbidity and Morbidity in an Epidemiologic Sample Arch Gen Psychiatry. 1992;49(4):282-288. doi:10.1001/archpsyc.1992.01820040034004

28. Lijster JM, Dierckx B, Utens EM, Verhulst FC, Zieldorff C, Dieleman GC, Legerstee JS. The Age of Onset of Anxiety Disorders Can J Psychiatry. 2017 Apr;62(4):237-246. doi: 10.1177/0706743716640757. Epub 2016 Jul 9. PMID: 27310233; PMCID: PMC5407545.

29. Fehm L, Beesdo K, Jacobi F, Fiedler A. Social anxiety disorder above and below the diagnostic threshold: prevalence, comorbidity, and impairment in the general population. Soc Psychiatry Psychiatr Epidemiol. 2008 Apr;43(4):257-65. doi: 10.1007/s00127-007-0299-4. Epub 2007 Dec 14. PMID: 18084686.

30. Nepon J, Belik SL, Bolton J, Sareen J. The relationship between anxiety disorders and suicide attempts: Findings from the National Epidemiologic Survey on Alcohol and Related Conditions. Depress Anxiety. 2010;27(9):791-8. doi: 10.1002/da.20674. PMID: 20217852 ; PMCID: PMC2940247.

31. Thibodeau MA, Welch PG, Sareen J, Asmundson GJ. Anxiety disorders are independently associated with suicide ideation and attempts: propensity score matching in two epidemiological samples. Depress Anxiety. 2013;30(10):947-54. doi: 10.1002/da.22203. PMID: 24108489.

32. Moitra E, Beard C, Weisberg RB. Occupational impairment and social anxiety disorder in a sample of primary care patients J Affect Disord. 2011;130(1-2):209-12. doi: 10.1016/j.jad.2010.09.024. Epub 2010 Oct 12. PMID: 20934220; PMCID: PMC3831271.

33. Wittchen HU, Zhao S, Kessler RC, Eaton WW. DSM-III-R generalized anxiety disorder in the National Comorbidity Survey. Arch Gen Psychiatry. 1994;51(5):355-64. doi: 10.1001/archpsyc.1994.03950050015002. PMID: 8179459.

34. Weisberg RB. Overview of generalized anxiety disorder: epidemiology, presentation, and course J Clin Psychiatry. 2009;70 Suppl 2:4-9. PMID: 19371500.

35. Wittchen HU, Hoyer J. Generalized anxiety disorder: nature and course. J Clin Psychiatry. 2001;62 Suppl 11:15-9; discussion 20-1. PMID: 11414546.

36. Li Y, Shi S, Yang F, Gao J, Li Y, Tao M, Wang G, Zhang K, Gao C, Liu L, Li K, Li K, Liu Y, Wang X, Zhang J, Lv L, Wang X, Chen Q, Hu J, Sun L, Shi J, Chen Y, Xie D, Flint J, Kendler KS, Zhang Z. Patterns of co-morbidity with anxiety disorders in Chinese women with recurrent major depression. Psychol Med. 2012;42(6):1239-48. doi: 10.1017/S003329171100273X. Epub 2011 Nov 30. PMID: 22126712; PMCID: PMC3339636. 
590

591

592

593

594

595

596

597

598

599

600

601

602

603

604

605

606

607

608

609

610

611

612

613

614

615

37. Preti A, Girolamo Gd, Vilagut G, Alonso J, Graaf Rd, Bruffaerts R, Demyttenaere K, Pinto-Meza A, Haro JM, Morosini P, ESEMeD-WMH Investigators. The epidemiology of eating disorders in six European countries: results of the ESEMeD-WMH project. J Psychiatr Res. 2009;43(14):1125-32. doi: 10.1016/j.jpsychires.2009.04.003. Epub 2009 May 8. PMID: 19427647.

38. LaSalle VH, Cromer KR, Nelson KN, Kazuba D, Justement L, Murphy DL. The diagnostic interview assessed neuropsychiatric disorder comorbidity in 334 individuals with obsessive-compulsive disorder. Depress Anxiety. 2004;19(3):163-73. doi: 10.1002/da.20009. PMID: 15129418.

39. Mathes BM, Henry A, Schmidt NB, Norberg MM. Hoarding symptoms and workplace impairment Br J Clin Psychol. 2019;58(3):342-356. doi: 10.1111/bjc.12212. Epub 2018 Dec 11. PMID: 30548281.

40. Joshi G, Wozniak J, Petty C, Vivas F, Yorks D, Biederman J, Geller D. Clinical characteristics of comorbid obsessive-compulsive disorder and bipolar disorder in children and adolescents. Bipolar Disord. 2010;12(2):185-95. doi: 10.1111/j.13995618.2010.00795.x. PMID: 20402711; PMCID: PMC2864069.

41. Kawakami N, Tsuchiya M, Umeda M, Koenen KC, Kessler RC. World Mental Health Survey Japan. Trauma and posttraumatic stress disorder in Japan: results from the World Mental Health Japan Survey. J Psychiatr Res. 2014;53:157-65. doi: 10.1016/j.jpsychires.2014.01.015. Epub 2014 Feb 6. PMID: 24572682; PMCID: PMC4169235.

42. Wang, X., Cheng, S. \& Xu, H. Systematic review and meta-analysis of the relationship between sleep disorders and suicidal behaviour in patients with depression. BMC Psychiatry. 2019;19:303. https://doi.org/10.1186/s12888-019-2302-5 
Table 1. Participant characteristics and evaluation scale scores

\begin{tabular}{|c|c|c|c|c|c|c|c|c|c|c|c|}
\hline & $\begin{array}{c}\text { Total } \\
n=1411\end{array}$ & $\begin{array}{c}\text { Mood } \\
\text { disorders } \\
\text { group } \\
\mathrm{n}=417\end{array}$ & $\begin{array}{l}\text { Control group } \\
n=994\end{array}$ & $p$-value & $\begin{array}{l}\text { Depression group } \\
\qquad \mathrm{n}=113\end{array}$ & $\begin{array}{c}\text { Control group } \\
n=1298\end{array}$ & $p$-value & $\begin{array}{l}\text { Dysthymia group } \\
\qquad n=41\end{array}$ & $\begin{array}{l}\text { Control group } \\
\qquad n=1370\end{array}$ & $p$-value & $\begin{array}{l}\text { Bipolar disorder group } \\
\qquad \mathrm{n}=353\end{array}$ \\
\hline \multicolumn{12}{|l|}{ Gender } \\
\hline Male (\%) & 1047 & $310(29.6)$ & $737(70.4)$ & & $79(7.5)$ & $968(92.5)$ & & $29(2.8)$ & $1018(97.2)$ & & $266(25.4)$ \\
\hline Female (\%) & 364 & $107(29.4)$ & $257(70.6)$ & 0.94 & $34(9.3)$ & $330(90.7)$ & 0.28 & $12(3.3)$ & $352(96.7)$ & 0.61 & $87(23.9)$ \\
\hline Age (years) & $40.1 \pm 10.3$ & $38.0 \pm 10.1$ & $41.0 \pm 10.3$ & $<0.001$ & $37.0 \pm 10.0$ & $40.4 \pm 10.3$ & $<0.005$ & $41.2 \pm 9.9$ & $40.1 \pm 10.3$ & 0.47 & $37.9 \pm 10.0$ \\
\hline $\begin{array}{l}\text { Consecutive } \\
\text { service(years) }\end{array}$ & $13.3 \pm 9.6$ & $12.3 \pm 9.2$ & $13.7 \pm 9.8$ & $<0.01$ & $12.7 \pm 9.4$ & $13.3 \pm 9.6$ & 0.51 & $16.9 \pm 10.5$ & $13.2 \pm 9.6$ & $<0.05$ & $12.1 \pm 9.1$ \\
\hline CES-D (points) & $14.1 \pm 8.3$ & $18.4 \pm 10.2$ & $12.3 \pm 6.6$ & $<0.001$ & $27.7 \pm 10.2$ & $12.9 \pm 6.9$ & $<0.001$ & $30.3 \pm 10.4$ & $13.6 \pm 7.7$ & $<0.001$ & $16.9 \pm 9.7$ \\
\hline BSDS (points) & $4.8 \pm 2.4$ & $6.0 \pm 3.2$ & $4.4 \pm 1.7$ & $<0.001$ & $6.8 \pm 3.8$ & $4.7 \pm 2.1$ & $<0.001$ & $6.7 \pm 4.0$ & $4.8 \pm 2.3$ & $<0.001$ & $6.0 \pm 3.2$ \\
\hline LSAS (points) & $39.9 \pm 28.2$ & $47.3 \pm 29.4$ & $36.7 \pm 27.1$ & $<0.001$ & $58.9 \pm 30.1$ & $38.2 \pm 27.4$ & $<0.001$ & $58.9 \pm 31.9$ & $39.3 \pm 27.9$ & $<0.001$ & $45.3 \pm 29.1$ \\
\hline SDISS1 (points) & $1.8 \pm 2.1$ & $2.8 \pm 2.4$ & $1.4 \pm 1.7$ & $<0.001$ & $4.5 \pm 2.5$ & $1.6 \pm 1.8$ & $<0.001$ & $5.4 \pm 2.5$ & $1.7 \pm 2.0$ & $<0.001$ & $2.5 \pm 2.3$ \\
\hline SDISS2 (points) & $1.6 \pm 2.0$ & $2.5 \pm 2.3$ & $1.2 \pm 1.6$ & $<0.001$ & $3.9 \pm 2.4$ & $1.4 \pm 1.8$ & $<0.001$ & $5.0 \pm 2.5$ & $1.5 \pm 1.9$ & $<0.001$ & $2.3 \pm 2.3$ \\
\hline SDISS3 (points) & $1.4 \pm 1.9$ & $2.1 \pm 2.3$ & $1.1 \pm 1.6$ & $<0.001$ & $3.2 \pm 2.7$ & $1.2 \pm 1.7$ & $<0.001$ & $4.0 \pm 2.8$ & $1.3 \pm 1.8$ & $<0.001$ & $2.1 \pm 2.2$ \\
\hline
\end{tabular}


Table 2. Group comparisons on MINI items

\begin{tabular}{|c|c|c|c|c|c|c|c|c|c|c|c|c|c|c|c|c|}
\hline & Total & $\begin{array}{c}\text { Mood } \\
\text { disorder } \\
\text { group }\end{array}$ & $\begin{array}{l}\text { Control } \\
\text { group }\end{array}$ & $\begin{array}{l}\text { Gross } \\
\text { odds } \\
\text { ratio }\end{array}$ & $\begin{array}{c}95 \% \\
\text { confidence } \\
\text { interval }\end{array}$ & $\begin{array}{c}\mathrm{p}- \\
\text { value }\end{array}$ & $\begin{array}{l}\text { Depression } \\
\text { group }\end{array}$ & $\begin{array}{l}\text { Control } \\
\text { group }\end{array}$ & $\begin{array}{l}\text { Gross } \\
\text { odds } \\
\text { ratio }\end{array}$ & $\begin{array}{c}95 \% \\
\text { confidence } \\
\text { interval }\end{array}$ & $\begin{array}{c}\mathrm{p}- \\
\text { value }\end{array}$ & $\begin{array}{l}\text { Dysthymia } \\
\text { group }\end{array}$ & $\begin{array}{l}\text { Control } \\
\text { group }\end{array}$ & $\begin{array}{l}\text { Gross } \\
\text { odds } \\
\text { ratio }\end{array}$ & $\begin{array}{c}95 \% \\
\text { confidence } \\
\text { interval }\end{array}$ & $\begin{array}{c}\mathrm{p}- \\
\text { value }\end{array}$ \\
\hline & $\mathrm{n}=1411$ & $\mathrm{n}=113$ & $n=1298$ & & & & $\mathrm{n}=113$ & $n=1298$ & & & & $\mathrm{n}=41$ & $\mathrm{n}=1370$ & & & \\
\hline A Depression & 113 & - & - & - & - & - & - & - & - & - & - & 23 & 90 & 18.2 & $9.5-34.9$ & $<0$. \\
\hline A1 & 64 & - & - & - & - & - & - & - & - & - & - & 21 & 43 & 32.4 & $16.4-64.2$ & $<0.001$ \\
\hline $\mathrm{A} 2$ & 88 & - & - & - & - & - & - & - & - & - & - & 15 & 73 & 10.3 & $5.2-20.2$ & $<0.001$ \\
\hline D Bipolar disorder group & 353 & - & - & - & - & - & 55 & 298 & 3.2 & $2.2-4.7$ & $<0.001$ & 21 & 332 & 3.3 & $1.8-6.1$ & $<0.001$ \\
\hline D1 High-spirited group & 218 & - & - & - & - & - & 28 & 190 & 1.9 & $1.2-3.0$ & $<0.01$ & 13 & 205 & 2.6 & $1.3-5.2$ & $<0.005$ \\
\hline D2 Increased irritability group & 216 & - & - & - & - & - & 40 & 176 & 3.5 & $2.3-5.3$ & $<0.001$ & 19 & 197 & 5.1 & $2.7-9.7$ & $<0.001$ \\
\hline E1 Panic disorder group & 45 & 10 & 35 & 9.0 & $4.4-18.4$ & $<0.001$ & 17 & 28 & 8.0 & $4.2-15.2$ & $<0.001$ & 7 & 38 & 7.2 & $3.0-17.3$ & $<0.0$ \\
\hline F1 Agoraphobia group & 150 & 55 & 95 & 5 & $3.5-7.2$ & $<0.001$ & 38 & 112 & 5.4 & $3.5-8.3$ & $<0.001$ & 11 & 139 & 5.4 & $1.6-6.6$ & $<0.0$ \\
\hline G1 Social anxiety disorder group & 142 & 92 & 50 & 5.3 & $3.7-7.7$ & $<0.001$ & 42 & 100 & 7.1 & $4.6-10.9$ & $<0.001$ & 16 & 126 & 6.3 & 3.3-12.1 & $<0.001$ \\
\hline O Generalized anxiety disorder group & 157 & 100 & 57 & 5.2 & $3.7-7.4$ & $<0.001$ & 49 & 108 & 8.4 & $5.5-12.9$ & $<0.001$ & 24 & 133 & 13.1 & $6.9-25.1$ & $<0.001$ \\
\hline H1 Obsession group & 71 & 53 & 18 & 7.9 & $4.6-13.7$ & $<0.001$ & 35 & 36 & 15.7 & $9.4-26.4$ & $<0.001$ & 19 & 52 & 21.9 & $11.2-42.9$ & $<0.001$ \\
\hline H2 Obsessive behavior group & 64 & 41 & 23 & 4.6 & $2.7-7.8$ & $<0.001$ & 17 & 47 & 4.7 & $2.6-8.5$ & $<0.001$ & 7 & 57 & 4.7 & $2.0-11.2$ & $<0.001$ \\
\hline I Post-traumatic stress disorder group & 204 & 96 & 108 & 2.5 & $1.8-3.3$ & $<0.001$ & 31 & 173 & 2.5 & $1.6-3.8$ & $<0.001$ & 12 & 192 & 2.5 & $1.3-5.1$ & $<0.01$ \\
\hline I1 Traumatic experience group & 201 & 93 & 108 & 2.4 & $1.7-3.2$ & $<0.001$ & 26 & 175 & 1.9 & $1.2-3.1$ & $<0.05$ & 9 & 192 & 1.7 & $0.8-3.7$ & 0.152 \\
\hline $\begin{array}{l}12 \text { Sense of powerlessness/fear } \\
\text { group }\end{array}$ & 98 & 56 & 42 & 3.5 & $2.3-5.3$ & $<0.001$ & 18 & 80 & 2.9 & $1.7-5.0$ & $<0.001$ & 6 & 92 & 2.4 & $1.0-5.8$ & $<0.05$ \\
\hline I3 Flashback group & 18 & 14 & 4 & 8.6 & $2.8-26.3$ & $<0.001$ & 12 & 6 & 25.6 & $9.4-69.6$ & $<0.001$ & 7 & 11 & 25.4 & $9.3-69.6$ & $<0.001$ \\
\hline J Alcohol misuse group & 726 & 240 & 486 & 1.4 & $1.1-1.8$ & $<0.005$ & 59 & 667 & 1.0 & $0.7-1.5$ & 0.87 & 20 & 726 & 0.9 & $0.5-1.7$ & 0.728 \\
\hline K Drug misuse group & 7 & 5 & 2 & 6.0 & $1.2-31.2$ & $<0.05$ & 4 & 3 & 15.8 & $3.5-71.7$ & $<0.001$ & 2 & 5 & 14 & $2.6-74.4$ & $<0.001$ \\
\hline
\end{tabular}




23

23

4.4

2.6-7.4

0.001

$63 \quad 22$

$\begin{array}{rr}41 & 1.3 \\ 129 & 3.8\end{array}$

17

$<0.001$

10
48

$\begin{array}{r}45 \\ 53 \\ 232 \\ \hline\end{array}$

MINI: Mini-International Neuropsychiatric Interview 


\begin{tabular}{|c|c|c|c|c|c|c|c|c|c|c|c|c|}
\hline & \multicolumn{3}{|c|}{ Mood disorders group } & \multicolumn{3}{|c|}{ Depression group } & \multicolumn{3}{|c|}{ Dysthymia group } & \multicolumn{3}{|c|}{ Bipolar disorder group } \\
\hline & $\begin{array}{c}\text { Adjusted } \\
\text { odds } \\
\text { ratio }\end{array}$ & $\begin{array}{c}95 \% \\
\text { confidence } \\
\text { interval }\end{array}$ & $p$-value & $\begin{array}{l}\text { Adjusted } \\
\text { odds } \\
\text { ratio }\end{array}$ & $\begin{array}{c}95 \% \\
\text { confidence } \\
\text { interval }\end{array}$ & $\mathrm{p}$-value & $\begin{array}{c}\text { Adjusted } \\
\text { odds } \\
\text { ratio }\end{array}$ & $\begin{array}{c}95 \% \\
\text { confidence } \\
\text { interval }\end{array}$ & $\mathrm{p}$-value & $\begin{array}{c}\text { Adjusted } \\
\text { odds } \\
\text { ratio }\end{array}$ & $\begin{array}{c}95 \% \\
\text { confidence } \\
\text { interval }\end{array}$ & $p$-value \\
\hline Panic disorder group & 3.19 & $1.42-7.17$ & $<0.05$ & 2.39 & $1.06-5.36$ & $<0.05$ & 1.94 & $0.62-6.02$ & 0.25 & 2.51 & $1.24-5.09$ & $<0.05$ \\
\hline Agoraphobia group & 2.10 & $1.36-3.23$ & $<0.01$ & 1.38 & $0.78-2.45$ & 0.28 & 0.55 & $0.21-1.44$ & 0.22 & 1.89 & $1.24-2.87$ & $<0.005$ \\
\hline Social anxiety disorder group & 2.62 & $1.71-4.02$ & $<0.001$ & 2.74 & $1.61-4.67$ & $<0.001$ & 2.16 & $0.92-5.05$ & 0.08 & 1.83 & $1.21-2.77$ & $<0.005$ \\
\hline Obsessive compulsive disorder group & 2.10 & $1.26-3.47$ & $<0.005$ & 3.59 & $2.02-6.38$ & $<0.001$ & 5.10 & $2.07-12.56$ & $<0.001$ & 1.16 & $0.71-1.90$ & 0,55 \\
\hline Post-traumatic stress disorder group & 1.69 & $1.20-2.39$ & $<0.005$ & 1.20 & $0.69-2.07$ & 0.52 & 1.07 & $0.44-2.60$ & 0.88 & 1.70 & $1.21-2.40$ & $<0.005$ \\
\hline Anorexia mentalis group & 1.13 & $0.62-2.06$ & 0.70 & 2.00 & $0.86-4.65$ & 0.11 & 3.33 & $1.01-11.00$ & $<0.05$ & 0.83 & $0.44-1.57$ & 0.57 \\
\hline Bulimia nervosa group & 2.78 & $2.04-3.78$ & $<0.001$ & 1.77 & $1.08-2.90$ & $<0.05$ & 1.33 & $0.59-3.02$ & 0.50 & 2.32 & $1.70-3.15$ & $<0.001$ \\
\hline Generalized anxiety disorder group & 1.75 & $1.14-2.69$ & $<0.05$ & 2.72 & $1.57-4.72$ & $<0.001$ & 6.97 & $3.01-16.16$ & $<0.001$ & 1.36 & $0.89-2.08$ & 0.15 \\
\hline Psychotic disorder group & 1.60 & $0.25-10.12$ & 0.62 & 5.09 & $0.85-30.56$ & 0.08 & 6.02 & $0.72-50.25$ & 0.10 & 0.67 & $0.11-3.97$ & 0.66 \\
\hline Drug misuse group & 1.77 & $0.93-3.38$ & 0.08 & 0.99 & $0.44-2.21$ & 0.98 & 0.64 & $0.20-2.06$ & 0.45 & 1.96 & $1.06-3.60$ & $<0.05$ \\
\hline Age & 0.99 & $0.98-1.00$ & 0.15 & 0.99 & $0.97-1.01$ & 0.40 & 1.05 & $1.01-1.08$ & 0.01 & 0.99 & $0.97-1.00$ & $<0.05$ \\
\hline Constant & 0.30 & & $<0.001$ & 0.05 & & $<0.001$ & 0.01 & & $<0.001$ & 0.33 & & $<0.001$ \\
\hline
\end{tabular}

